\title{
Out of the darkness: the infrared afterglow of the INTEGRAL burst GRB 040422 observed with the VLT ${ }^{\star \star}$
}

\author{
P. Filliatre ${ }^{1,2}$, P. D’Avanzo ${ }^{3,4}$, S. Covino ${ }^{5}$, D. Malesani ${ }^{6}$, G. Tagliaferri ${ }^{5}$, S. McGlynn ${ }^{7}$, L. Moran ${ }^{8}$, P. Goldoni ${ }^{2,1}$, \\ S. Campana ${ }^{5}$, G. Chincarini ${ }^{5,4}$, L. Stella ${ }^{9}$, M. Della Valle ${ }^{10}$, N. Gehrels ${ }^{11}$, S. McBreen ${ }^{12}$, L. Hanlon ${ }^{7}$, B. McBreen ${ }^{7}$, \\ J. A. Nousek ${ }^{13}$, and R. Perna ${ }^{14}$
}

${ }^{1}$ Laboratoire Astroparticule et Cosmologie, UMR 7164, 11 place Marcelin Berthelot, 75231 Paris Cedex 05, France e-mail: filliatr@cea.fr

2 Service d'Astrophysique, CEA/DSM/DAPNIA/SAp, CE-Saclay, Orme des Merisiers, Bât. 709, 91191 Gif-sur-Yvette Cedex, France

3 Università dell'Insubria, Dipartimento di Fisica e Matematica, via Vallegio, 11, 22100 Como, Italy

${ }^{4}$ Università degli studi di Milano-Bicocca, Dipartimento di Fisica, Piazza delle Scienze 3, 20126 Milano, Italy

5 INAF, Osservatorio Astronomico di Brera, via E. Bianchi 46, 23807 Merate (LC), Italy

${ }^{6}$ International school for advanced studies (SISSA/ISAS), via Beirut 2-4, 34014 Trieste, Italy

7 Department of Experimental Physics, University College, Dublin 4, Ireland

8 Department of Physics \& Astronomy, University of Southampton, Southampton, SO17 1BJ, UK

9 INAF, Osservatorio Astronomico di Roma, via Frascati 33, Monteporzio Catone, 00040 Rome, Italy

${ }_{10}$ INAF - Osservatorio Astrofisico di Arcetri, largo E. Fermi 5, 50125 Firenze, Italy

11 NASA Goddard Space Flight Center, Code 661, Greenbelt, MD 20771, USA

12 Astrophysics Missions Division, Research Scientific Support Department of ESA, ESTEC, Noordwijk, The Netherlands

13 Department of Astronomy and Astrophysics, Pennsylvania State University, 525 Davey Laboratory, University Park, PA 16802, USA

14 Department of Astrophysical and Planetary Sciences, University of Colorado at Boulder, 440 UCB, Boulder, CO, 80309, USA

Received 23 December 2004 / Accepted 31 March 2005

\begin{abstract}
GRB 040422 was detected by the INTEGRAL satellite at an angle of only 3 degrees from the Galactic plane. Analysis of the prompt emission observed with the SPI and IBIS instruments on INTEGRAL are presented. The IBIS spectrum is well fit by the Band model with a break energy of $E_{0}=56 \pm 2 \mathrm{keV}$ and $E_{\text {peak }}=41 \pm 3 \mathrm{keV}$. The peak flux is $1.8 \times 10^{-7} \mathrm{erg} \mathrm{cm}^{-2} \mathrm{~s}^{-1}$ and fluence $3.4 \times 10^{-7} \mathrm{erg} \mathrm{cm}^{-2}$ in the range $20-200 \mathrm{keV}$. We then present the observations of the afterglow of GRB 040422 , obtained with the ISAAC and FORS 2 instruments at the VLT less than $2 \mathrm{~h}$ after the burst. We report the discovery of its near-infrared afterglow, for which we give the astrometry and photometry. No detection could have been obtained in the $R$ and $I$ bands, partly due to the large extinction in the Milky Way. We imaged the position of the afterglow again two months later in the $K_{\mathrm{s}}$ band, and detected a likely bright host galaxy. We compare the magnitude of the afterglow with those of a compilation of promptly observed counterparts of previous GRBs, and show that the afterglow of GRB 040422 lies at the very faint end of the distribution, brighter only than that of GRB 021211, singled out later and in the optical bands, and GRB 040924 after accounting for Milky Way extinction. This observation suggests that the proportion of dark GRBs can be lowered significantly by a more systematic use of $8-\mathrm{m}$ class telescopes in the infrared in the very early hours after the burst.
\end{abstract}

Key words. gamma rays: bursts

\section{Introduction}

* Based on observations with INTEGRAL, an ESA project with instruments and science data centre funded by ESA member states (especially the PI countries: Denmark, France, Germany, Italy, Switzerland, Spain), Czech Republic, and Poland, and with the participation of Russia and the USA.

$\star \star$ Based on observations made with ESO telescopes at the Paranal Observatory under programme 073.D-0255.
The study of GRB afterglows is a promising tool for cosmology, as their absorption spectra convey information on the distance and the chemical environment of a new set of galaxies (e.g. Fiore et al. 2004), with the possibility of exploration up to the reionization epoch (Lamb \& Reichart 2000). However, a debated fraction of GRBs - from less than $10 \%$ (Lamb et al. 2004) to 60\% (Lazzati et al. 2002) - did not 
show any detectable afterglow in the optical band. Popular and non-mutually exclusive explanations are: these bursts have intrinsically faint afterglows in the optical band (e.g. Fynbo et al. 2001; Lazzati et al. 2002); their decay is very fast (Berger et al. 2002); the optical afterglow is extinguished by dust in the vicinity of the GRB or in the starforming region in which the GRB occurs (e.g. Lamb 2000; Reichart \& Price 2002); their redshift is above 6, so that the Lyman- $\alpha$ absorption by neutral hydrogen in the host galaxy and along the line of sight damps the optical radiation of the afterglow (Lamb 2000). To these physical explanations, one must add the possibility that the search techniques are neither accurate nor quick enough (Lamb et al. 2004). The possibility that some afterglows are intrinsically faint has of course the biggest impact on the modelling of the GRBs, as well as on their application in cosmology. On the other hand, if one or several of the other explanations are correct, a substantial reduction of the fraction of dark bursts can be achieved by quick observations in the infrared.

For this reason, we adopted a strategy to promptly search for GRB counterparts both in the near-infrared (NIR) and in the optical. To date, several attempts have been performed, sometimes leading to a detection (as for GRB 040827: Malesani et al. 2004c) and sometimes just to upper limits (as for GRB 040223: Tagliaferri et al. 2004). This paper deals with the observations of GRB 040422 performed with the ISAAC and FORS 2 instruments installed at the focal plane of the Very Large Telescope (VLT) at the European Southern Observatory (ESO). In this case, a positive detection of the afterglow was nicely achieved.

ESA's International Gamma-Ray Astrophysics Laboratory INTEGRAL (Winkler et al. 2003), launched in October 2002, is composed of two main telescopes, an imager IBIS (Imager on Board the INTEGRAL Satellite, Ubertini et al. 2003), and a spectrometer SPI (Spectrometer on INTEGRAL, Vedrenne et al. 2003) coupled with two monitors, one in the $\mathrm{X}$-ray band and one in the optical band. Although not built as a dedicated GRB-mission, INTEGRAL has a burst alert system called IBAS (INTEGRAL Burst Alert System, Mereghetti et al. 2003). IBAS carries out rapid localizations for GRBs incident on the IBIS detector with a precision of a few arcminutes (Mereghetti et al. 2004a). The public distribution of these coordinates enables multi-wavelength searches for afterglows at lower energies. INTEGRAL data on the prompt emission in combination with the early multi-wavelength studies, such as presented in this work, can probe these high energy phenomena.

GRB 040222 was detected by INTEGRAL on April 22, 2004 at 06:58:02 UTC (Apr 22.290) with the IBIS/ISGRI instrument in the 15-200 keV band (Mereghetti et al. 2004b). It was localized with coordinates $\alpha=18^{\mathrm{h}} 42^{\mathrm{m}} 00^{\mathrm{s}}, \delta=+01^{\circ} 59^{\prime} 29^{\prime \prime}$ (J2000.0) with an uncertainty radius of $2.5^{\prime}$. We will hereafter refer to this localization as the IBAS error box. The reported peak flux in the $20-200 \mathrm{keV}$ range is about 2.7 photons $\mathrm{cm}^{-2} \mathrm{~s}^{-1}$ $\left(2.5 \times 10^{-7} \mathrm{erg} \mathrm{cm}^{-2} \mathrm{~s}^{-1}\right)$. In this paper we present the spectral analysis that was performed with the spectrometer SPI and imager IBIS.
The counterpart was very promptly sought by ROTSE, which found no new object up to $R \sim 16.5$ less than $30 \mathrm{~s}$ after the burst (Rykoff 2004), although field crowding creates significant source confusion. We carried out our search for the afterglow in the $R, I$, and $K_{\mathrm{s}}$ bands very quickly within $2 \mathrm{~h}$ after the burst. Lacking a refined X-ray or radio position, the entire IBAS error box was explored. No afterglow candidate was detected in the error box down to the limit $K=14.5$ when compared with the 2 MASS catalog (Malesani et al. 2004a). In this paper, we report the discovery of a faint NIR afterglow with a refined off-line analysis. Subsequent observations carried out 64 days after the burst revealed the presence of a likely host galaxy. We present these results in the context of dark or optically faint bursts and discuss the advantage of rapid follow-up observations in the infrared.

\section{Prompt gamma ray emission with INTEGRAL}

IBIS and SPI are coded mask instruments, and the photons from a single point source are spread over all the individual detectors. Spectral extraction is possible using specifically designed software which consists of modelling the illuminated mask by a point source of unitary flux placed at the sky coordinates of the GRB. The model is then fit to the detected shadowgram in each channel to obtain the rate and error for each channel.

GRB 040422 was visible in the partially coded field of view ( $9.4^{\circ}$ off-axis) of both IBIS and SPI. The burst was outside the field of view of the two monitoring instruments, JEM-X (Joint European X-Ray Monitor) and OMC (Optical Monitoring Camera). This GRB falls into the class of long bursts with a duration $T_{90}=4 \mathrm{~s}$ obtained from the IBIS light curve. The GRB was localized at $\alpha=18^{\mathrm{h}} 42^{\mathrm{m}} 01^{\mathrm{s}} .2, \delta=+1^{\circ} 59^{\prime} 01^{\prime \prime} .6$ (J2000.0) in the IBIS data with an error radius of $1.7^{\prime}$. Note that this localization was obtained by an off-line analysis that was not available when we triggered our VLT observations, so that it was not used for the search of the afterglow. It is nevertheless fully consistent with the IBAS error box of 2.5' we used for this purpose. The position of the burst, extracted from the SPI data in the energy range $20-100 \mathrm{keV}$ with $S / N=11.7$, is $\alpha=18^{\mathrm{h}} 42^{\mathrm{m}} 00^{\mathrm{s}} .7$, $\delta=+1^{\circ} 53^{\prime} 13^{\prime \prime}$ '2 (J2000.0) which is $6^{\prime}$ away from the IBIS location, so consistent with the $10^{\prime}$ location accuracy of SPI for a source with $S / N \sim 10$.

\subsection{SPI analysis}

Determination of SPI light curves with binning on a short time scale is possible only using the $1 \mathrm{~s}$ count rates of the germanium detectors, which are usually used for scientific housekeeping purposes. These values reflect the count rates of each detector in the broad SPI energy band from $20 \mathrm{keV}$ to $8 \mathrm{MeV}$. The SPI light curve in Fig. 1 was generated by summing the background-subtracted rates. The background was determined from a 40-min period before the burst trigger and was subtracted from each detector individually. The SPI light curve is a single pulse that does not have the FRED profile that is observed in many singled-peaked bursts and in approximately $7 \%$ of BATSE bursts (Fishman \& Meegan 1995). 


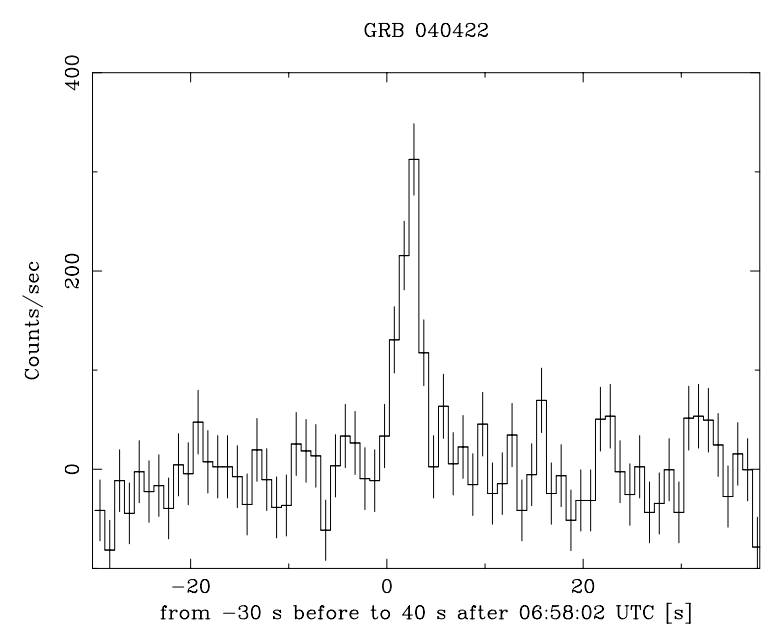

Fig. 1. Light curve of GRB 040422 relative to the IBAS trigger extracted from SPI with timing resolution of $1 \mathrm{~s}$ from $20 \mathrm{keV}$ to $8 \mathrm{MeV}$.

A spectrum was extracted for one time interval of $4 \mathrm{~s}$ starting at 06:58:02 UTC. All single events detected by SPI, corrected for intrinsic deadtimes and telemetry gaps, were binned into 5 equally spaced logarithmic energy bins in the $20-200 \mathrm{keV}$ range for the 4-s duration of the burst. Version 4.1 of the OSA from the INTEGRAL Science Data Centre (Courvoisier et al. 2003) and the software package SPIROS 8 (Skinner \& Connell 2003) were used for SPI spectral extraction, while XSPEC 11.2 was used for model fitting. The background was determined from a 40-min period of SPI data before the burst trigger, as for the light curve. The spectrum obtained is best fit by a single power-law model yielding a photon index $\Gamma=-2.17_{-0.28}^{+0.24}$ in the energy range $20-200 \mathrm{keV}$, with a reduced $\chi^{2}$ of 2.5 with 3 degrees of freedom, while the errors are quoted for 1 parameter of interest at the $90 \%$ confidence level. The peak flux obtained in the same energy range is 2.8 photons $\mathrm{cm}^{-2} \mathrm{~s}^{-1}$. The same analysis was performed for multiple events detected by the SPI detectors, but yielded no significant improvement to the fit. The Band model (Band et al. 1993) was also fit to the SPI data for GRB 040422 yielding a low value for the break energy $E_{0}=60 \pm 21 \mathrm{keV}$. However there is a low probability $(0.44, f$-test $)$ that the addition of extra model components improves the fit over a single power-law.

\subsection{IBIS analysis}

The data were obtained from the ISGRI detector of IBIS, which is an array of $128 \times 128 \mathrm{CdTe}$ crystals sensitive to lower-energy gamma-rays (Lebrun et al. 2003), and were used to produce the light curves of GRB 040422. The IBIS time profile of the GRB is given in Fig. 2 for the energy ranges $15-40$ and $40-200 \mathrm{keV}$ with a time resolution of $0.25 \mathrm{~s}$. On this time scale, the GRB is resolved into two pulses with the second pulse harder and more intense than the first. A spectrum was extracted for one time interval of $4 \mathrm{~s}$ starting at 06:58:02 UTC. The spectrum was fit by a single power-law model with a photon index of $\Gamma=-2.2 \pm 0.4$ and with a reduced $\chi^{2}$ of 2.4 for 36 degrees of freedom, consistent with the SPI determination. The Band
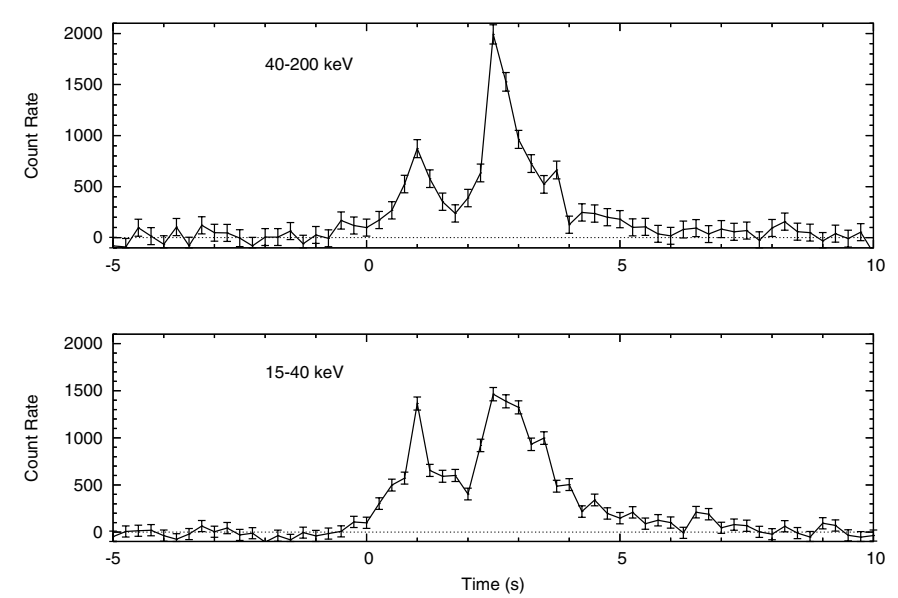

Fig. 2. Light curves of GRB 040422 extracted from IBIS/ISGRI data in the energy ranges 15-40 keV (top) and 40-200 keV (bottom) with $0.25 \mathrm{~s}$ timing resolution.

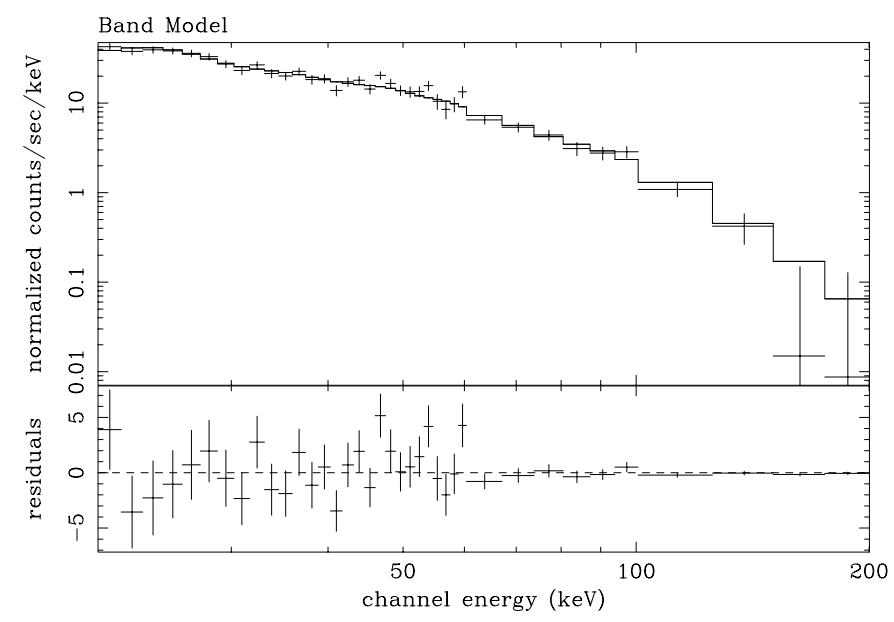

Fig. 3. IBIS/ISGRI spectrum of GRB 040422 fitted by a Band model from 20-200 keV. Upper panel: data and best fit model. Lower panel: residuals between the data and the folded model.

model gave a better fit to the IBIS data and yielded values of the break energy, $E_{0}=56 \pm 2 \mathrm{keV}$, photon index below the turnover $\alpha=-1.26 \pm 0.03$ (hence the peak energy is $E_{\text {peak }} \equiv(\alpha+2) E_{0}=$ $41 \pm 3 \mathrm{keV}$ ) and photon index above the turnover $\beta$ fixed at -4.0 where the errors quoted are for 1 parameter of interest at the $90 \%$ confidence level (Fig. 3). This fit resulted in a reduced $\chi^{2}$ of 1.14 for 35 degrees of freedom. The break energy $E_{0}$ is in the middle of the energy band where there is good sensitivity. The peak flux obtained for the Band model in the range $20-200 \mathrm{keV}$ is $2.3_{-0.5}^{+0.3}$ photons $\mathrm{cm}^{-2} \mathrm{~s}^{-1}\left(1.8_{-0.6}^{+0.3} \times 10^{-7} \mathrm{erg} \mathrm{cm}^{-2} \mathrm{~s}^{-1}\right)$, and the fluence in the same energy range is $3.4_{-1.0}^{+1.3} \times 10^{-7} \mathrm{erg} \mathrm{cm}^{-2}$.

\section{Observations with the VLT}

Table 1 shows the log of observations. Due to the size of the error box compared with the ISAAC field of view $\left(2.5^{\prime} \times 2.5^{\prime}\right)$, a mosaic of four panels was necessary in the NIR. In the optical, however, just one exposure was sufficient to cover the error box. The raw frames were processed using the standard procedure: subtraction of an averaged bias frame, division by a normalised flat frame, and, for 
Table 1. Log of the observation for GRB 040422. Observing times refer to the middle of exposures. Upper limits refer to a non-detection at $1 \sigma$ level with a confidence level of $95 \%$.

\begin{tabular}{lllllll}
\hline \hline $\begin{array}{l}\text { Date } \\
\text { (UTC) }\end{array}$ & $\begin{array}{l}\text { Time } \\
\text { since burst }\end{array}$ & $\begin{array}{l}\text { Seeing FWHM } \\
\prime\end{array}$ & Number $\times$ exposure time & Instrument & Filter & Magnitude \\
\hline 2004 Apr. 22.37 & $1.90 \mathrm{~h}$ & 0.9 & $5 \times 30 \mathrm{~s}$ & VLT+ISAAC & $K_{\mathrm{s}}$ & $18.0 \pm 0.1$ \\
2004 Apr. 22.37 & $1.93 \mathrm{~h}$ & 0.9 & $1 \times 120 \mathrm{~s}$ & VLT+FORS 2 & $R$ & $>24.2$ \\
2004 Apr. 22.38 & $2.09 \mathrm{~h}$ & 1.1 & $1 \times 120 \mathrm{~s}$ & VLT+FORS 2 & $I$ & $>23.4$ \\
2004 May 05.33 & 13.04 days & 1.8 & $5 \times 30 \mathrm{~s}$ & VLT+ISAAC & $K_{\mathrm{s}}$ & $>20.2$ \\
2004 Jun. 26.11 & 64.82 days & 0.8 & $30 \times 60 \mathrm{~s}$ & VLT+ISAAC & $K_{\mathrm{s}}$ & $20.3 \pm 0.2$ \\
\hline
\end{tabular}

the $K_{\mathrm{s}}$ frames, subtraction of a sky model obtained by taking the median of the dithered frames, followed by positionally registering the frames before co-adding them. Astrometry was performed using the GSC- 2 catalog ${ }^{1}$ for the FORS 2 frames and the $2 \mathrm{MASS}^{2}$ for the ISAAC frames. In the latter case, about 100 stars per panel were available, leading to an rms of 0.20 . The photometric measurements were made with SExtractor (Bertin \& Arnouts 1996) for the full catalogs and with $I R A F^{3}$ daophot task for single objects of interest. The calibration was done against the standard field CS 62 (Graham 1982) for FORS 2, and against the standard star S808-C (Persson et al. 1998) for ISAAC. In the NIR, a -0.2 mag correction was made for consistency with the 2 MASS catalog, using all the available stars of this catalog with $10 \leq K_{\mathrm{s}} \leq 15$ ( $\sim 50$ stars per panel) and checking that the results are robust with respect to this choice of magnitude range.

A mosaic of the four panels is shown in Fig. 4, covering $\sim 81 \%$ of the IBAS error box and showing $\sim 6 \times 10^{3}$ objects within it. For comparison we used the frames of the second epoch, which is significantly deeper than the 2MASS cata$\log$. We will assume throughout the paper that the afterglow light curve can be simply modelled by $F(t, v) \propto t^{-\alpha} v^{-\beta}$ (e.g. van Paradjis et al. 2000); that is, assuming a temporal index $\alpha$ of 1 with no jet break, we expect a magnitude difference of about 5.5 between the first and second epoch. As the seeing was poorer during our observations in the second epoch, we degraded the images of the first epoch (with psfmatch task of $I R A F$ ) accordingly. After aligning the first and second epoch frames, we used the SExtractor software to derive the list of detected sources at $2 \sigma$ and $1 \sigma$ confidence levels for the first and second epochs, respectively.

We set the following criteria to identify the potential afterglow candidates:

1) the object is seen in the first epoch; its $F W H M$ is larger than 0.7 ; its ellipticity is lower than 0.5 (as we are looking for a stellar-like object); and:

\footnotetext{
${ }^{1}$ http://www-gsss.stsci.edu/gsc/gsc2/GSC2home.htm

2 http://irsa.ipac.caltech.edu/

${ }^{3}$ IRAF is distributed by the National Optical Astronomy Observatories, which are operated by the Association of Universities for Research in Astronomy, Inc. under cooperative agreement with the National Science Foundation.
}

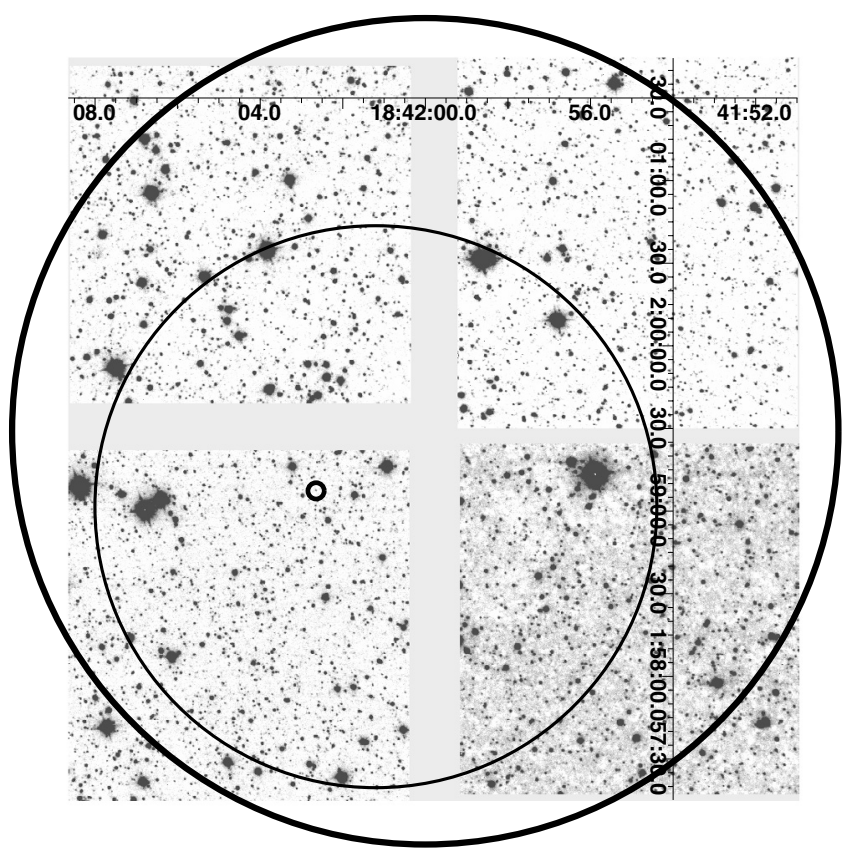

Fig. 4. A mosaic of the four panels observed by ISAAC. The large circle corresponds to the IBAS error box, the smaller thin one to the IBIS localization. The smallest circle is centred on our identification of the afterglow. North is up, East is left.

2a) no object is seen within a 0.3 radius in the second epoch (i.e. $1.5 \sigma$ of the astrometric error), or

2b) an object is seen at the right position, but is at least 2 magnitudes fainter, which would correspond to a temporal index of 0.36 , an unusually low value.

The number of potential candidates after this step is about 60 for each panel. The choice of different thresholds for the first and second epochs has helped to lower the number of false detections. We subsequently performed a careful visual inspection to eliminate spurious candidates:

- cosmic rays;

- double stars separated in the first epoch, but blended in the second epoch;

- objects blended with a close bright object;

- objects close to a bad pixel region;

- diffuse objects;

- objects that can be glimpsed in the second epoch, but escaped detection because their fluxes were below threshold. 
Only one candidate remains after this careful inspection. It is isolated, and stars with similar magnitudes are well above the detection threshold on the reference frame. Its coordinates are: $\alpha=18^{\mathrm{h}} 42^{\mathrm{m}} 02^{\mathrm{s}} .65, \delta=01^{\circ} 59^{\prime} 07^{\prime \prime} \cdot 35$ (J2000.0), i.e. $45^{\prime \prime}$ off the centre of the IBAS error box, and $22^{\prime \prime} 5$ off the centre of the IBIS error box reported in Sect. 2.2. Its $K_{\mathrm{s}}$ magnitude at the first epoch is $K_{\mathrm{s}}=18.0 \pm 0.1$, as reported in Table 1. This candidate is not detected in the second epoch frame. Careful analysis of the $R$ and $I$ frames yielded only upper limits given in Table 1.

In order to confirm the candidate afterglow we made a third set of observations with ISAAC about two months after the GRB and with a much longer integration time. This observation revealed, at the position of the candidate, a faint object with ellipticity of $\sim 0.7$, which spans $\sim 2.5^{\prime \prime}$ in its largest dimension. Its magnitude is $K_{\mathrm{s}}=20.3 \pm 0.2$, which corresponds to a $2 \sigma$ detection, the $1 \sigma$ detection limit being $K_{\mathrm{s}}=21$ (95\% C.L.). Over the $\sim 400$ objects detected in the range $20<K_{\mathrm{s}}<21$, only $0.7 \%$ have a greater ellipticity, indicating that it is unlikely that this object is a variable star. The possibility of a QSO has also been considered. The number of QSOs with $B<21$ is about 100 per square degree (Ulrich et al. 1997); hence, we can expect 0.5 in the IBAS error box. However, only a small fraction of these objects, the Optically Violently Variable (OVVs, or BL Lac), may have a variation of more than $2 \mathrm{mag}$ in 13 days, to comply with our observations in the first and second epochs. The amplitude of variability is expected to depend only weakly on the wavelength. The OVVs are not objects that have bursts and become very faint between two bursts, actually they have an erratic behaviour between two extrema that can reach $5 \mathrm{mag}$ or more. An estimate of the number of these objects is given by Collinge et al. (2004), as about 0.15 per square degree. The probability of finding such an object by chance in the IBAS error box is therefore very small, about $0.1 \%$. Then, although a firm rejection of this hypothesis cannot be made because of the scarcity of data, it seems very unlikely that the object is an OVV. We therefore suggest that this object is the host galaxy of GRB 040422. This deep observation strengthens our identification of the afterglow of GRB 040422.

A close-up around the afterglow position for the three epochs is shown in Fig. 5, and the light curve in Fig. 6. Using the first two epochs, we can constrain the temporal index to be $\alpha>0.4$, but this could be an average between two regimes in case of a jet break. This is consistent with the decay of observed afterglows, which have $\alpha$ in the range 0.7-2 (van Paradjis et al. 2000). Assuming a typical decay with $\alpha=1$, the contribution of the afterglow at the third epoch is negligible. Also, any associated supernova is not expected to contribute significantly at this late time. Conversely, the contribution of the host galaxy to the afterglow flux during the first epoch is less than $0.2 \mathrm{mag}$. We also note that the upper limit of ROTSE (Rykoff 2004) would translate to $R>22.4$ in our first epoch for $\alpha=1$, or that the ROTSE limit and our limit are equivalent within the power-law framework for $\alpha=1.3$. Such values for $\alpha$ are within the usual range.

The magnitudes reported in Table 1 are the observed values, and they do not take into account the absorption due to the Milky Way. The afterglow lies towards the Galactic

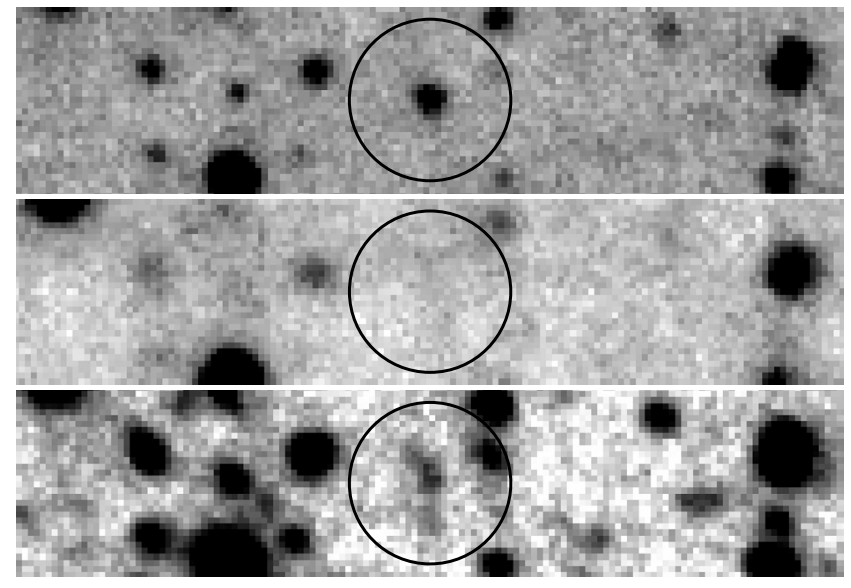

Fig. 5. The region of the candidate afterglow. From top to bottom: 2004 Apr. 22.37, 2004 May 05.33, 2004 Jun. 26.11. The circle around the afterglow on the first epoch has a radius of $2^{\prime \prime}$ to give the scale. The second epoch is affected by bad seeing. A logarithmic scale was chosen for the third epoch to bring out the elongated shape of the likely host galaxy.

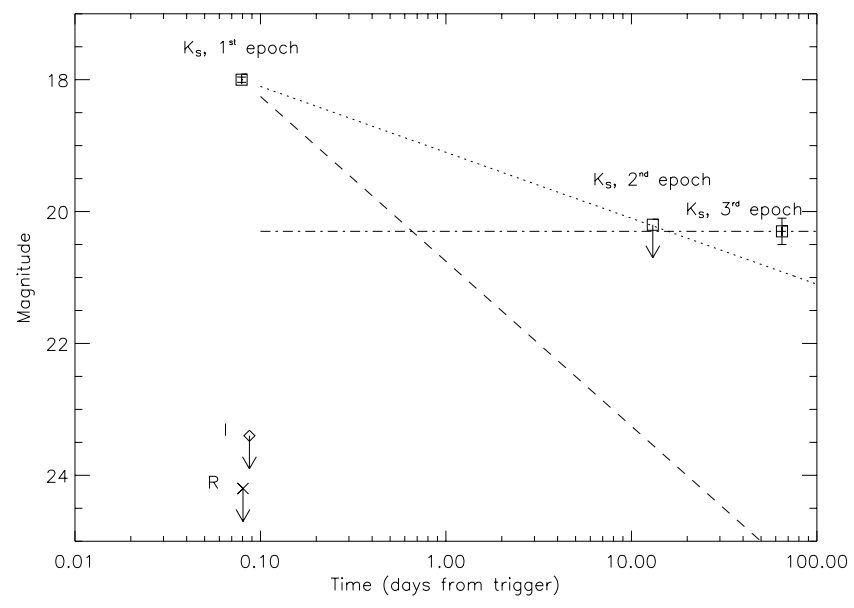

Fig. 6. The light curve of the afterglow of GRB 040422 . Two powerlaw models are included, with temporal indices of $\alpha=0.4$ (dotted line) and $\alpha=1$ (dashed line). The horizontal line gives the magnitude of the host.

plane, rather close to the direction of the Galactic centre ( $l=33^{\circ} 37^{\prime} 51^{\prime \prime} 85, b=2^{\circ} 59^{\prime} 41^{\prime \prime}, 83$ ), and this close to the Galactic plane, the extinction is high, rather patchy, and uncertain. The hydrogen column density is estimated to be $N_{\mathrm{H}}=$ $6 \times 10^{21} \mathrm{~cm}^{-2}$ (Dickey \& Lockman 1990). Using the fit of Predehl \& Schmitt (1995), we derive $A_{\mathrm{V}}=3.6$; and hence, using the extinction law of Cardelli et al. (1989) with $R_{\mathrm{V}}=3.1$, we get $E(B-V)_{N_{\mathrm{H}}}=1.17$. The use of the dust emission maps at $100 \mu \mathrm{m}$ by FIRAS obtained by Schlegel et al. (1998) leads to a substantially higher absorption of $E(B-V)_{\mathrm{FIR}}=1.92$, although according to the authors, the calculations should not be trusted for Galactic latitudes below $5^{\circ}$. This overestimate of the reddening has been considered by Dutra et al. (2003), who proposed a linear correction: $E(B-V)=0.748 E(B-V)_{\mathrm{FIR}}+0.0056$, valid for $0.7<E(B-V)<1$.6. In our case, we get $E(B-V)=$ 1.43 (thus in the validity domain of the relation), and then $A_{V}=4.4, A_{R}=3.8, A_{I}=2.8$ and $A_{K}=0.5$. In the rest of 
the paper, we will keep these values for definiteness. As expected, infrared observations are mandatory when looking for afterglows within the Galactic plane where the majority of the INTEGRAL alerts are concentrated. We can use these absorption values to constrain the power-law spectral index $\beta$ using our $R$ and $I$ upper limits combined with our $K_{\mathrm{s}}$ measurement (first epoch). As the frames were acquired almost simultaneously, the temporal evolution has little effect. We get $\beta>1.7$; conversely, if we suppose the more common value of $\beta=0.7$, we derive $E(B-V)>2.96$. This rather high value for $\beta$ might indicate the presence of an additional, intrinsic reddening or that there is Lyman- $\alpha$ damping, although the safer explanation is that our estimate of the Milky Way absorption is inaccurate. We note anyway that an afterglow with such a steep spectral index goes naturally undetected in the $R$ band where most searches are done, while it is much more easily detected in the $K_{\mathrm{s}}$ band. In other words, the observed distribution of $\beta$ is biased towards low values.

\section{Discussion}

\subsection{The prompt gamma ray emission: comparison with other INTEGRAL bursts}

Twenty GRBs occurred in the field of view of the main INTEGRAL instruments up to the end of 2004. One of these events, initially classified as a GRB (GRB 040903) is believed to be an X-ray flash (XRF) or a possible Type I X-ray flare from a new transient source in the Galactic bulge (Götz et al. 2004). In a second case, GRB 031203, modelling of the dustscattered X-ray echo provided the first proof of a low luminosity, XRF source (Vaughan et al. 2004; Watson et al. 2004). The IBIS spectrum, however, is consistent with a single power-law of photon index $-1.63 \pm 0.06$ (Sazonov et al. 2004; Soderberg et al. 2004), typical of INTEGRAL bursts. This event is the only INTEGRAL burst to date for which a direct redshift $(z=0.1055 \pm 0.0001$, Prochaska et al. 2004) measurement has been made.

A single power-law model with photon index in the range -1 to -2 provides a good fit to the data for the vast majority of INTEGRAL bursts in the range 20 to $200 \mathrm{keV}$ (e.g. von Kienlin et al. 2003; Moran et al. 2005; Mereghetti et al. 2004c). However, the SPI spectral index of GRB 040422 is very steep and lies outside this range for a power-law fit with $\Gamma=-2.17_{-0.28}^{+0.24}$. Prior to this burst the steepest photon index for an INTEGRAL burst was $\Gamma=-1.90 \pm 0.15$ for GRB 040403 (Mereghetti et al. 2004c). In addition, prior to GRB 040422, GRB 030131 was the only burst that was best fit by a Band model over its whole duration, with a break energy $E_{0}$ of $70 \pm 20 \mathrm{keV}$, a photon index below the turnover $\alpha$ of $-1.4 \pm 0.2$, and photon index above the turnover $\beta$ of $-3.0 \pm 1.0$ (Götz et al. 2003). GRB 040422 and GRB 030131 have similar spectral properties. The temporal properties of a large sample of bursts detected by the BATSE experiment are available (Quilligan et al. 2002; McBreen et al. 2002), and GRB 040422 is consistent with this sample. There is nothing to indicate that this burst is anything other than a normal cosmological GRB.

\subsection{The afterglow and the host galaxy}

The data that we presented here are the only ones available to date for the afterglow of GRB 040422 with a positive detection. They are rather scarce, the main reason being that because of the localization of the burst within the Galactic plane, the afterglow had not been localized quickly enough to undertake variability and spectroscopic studies. It is interesting to note that the host galaxy $\left(K_{\mathrm{s}}=19.8 \pm 0.2\right.$ after correction for the Milky Way reddening) is rather bright for a GRB host galaxy. In the sample of 19 host galaxies of Le Floc'h et al. (2004) (with $0.43 \leq z \leq 3.42$ and $19.05 \leq K \leq 23.5$ ), only two are brighter: the host of GRB 010921 with $K_{\mathrm{s}}=19.05 \pm 0.1$ and $z=0.45$, and the host of GRB 980703 with $K_{\mathrm{s}}=19.6 \pm 0.1$ and $z=0.97$. This might suggest that the galaxy has a rather small redshift. However, the strong Galactic absorption will prevent observations in the optical, and photometric observations in $J$, $H, K_{\mathrm{s}}$ will probably be insufficient for a photometric redshift if we face a starburst galaxy. Infrared spectroscopy is difficult and time-consuming even for the VLT; about a whole night would be needed for ISAAC to get a sufficient signal to noise ratio. We are facing a case where an early spectrum of the GRB afterglow would have been possibly the only way to securely derive the redshift.

We compared the afterglow of GRB 040422 with those of GRBs listed in Table 2 selected according to the following criteria:

- a photometric measurement of the optical counterpart were made in the first $24 \mathrm{~h}$ with good observing conditions; this timing constraint had two motivations: first, to avoid a bias towards bright afterglows that late detections tend to select; second, to avoid introducing a large error in extrapolating to the first epoch of our observations;

- temporal index $\alpha$ is available to extrapolate to the first epoch of our observations of GRB 040422 assuming that the lightcurve can be modelled by $F(t, v) \propto t^{-\alpha} v^{-\beta}$; in case of a temporal break, only the index before the break is adopted;

- the $K_{\mathrm{s}}$ magnitude is given, or the spectral index $\beta$ is given so that the extrapolation to this band can be made.

Using the spectral index, we extrapolate their magnitude to the $K_{\mathrm{s}}$ band and correct for absorption in the Milky Way (negligible for our purpose except for GRB 031203), using, as for GRB 040422, the $E(B-V)$ values from the FIRAS maps corrected according to Dutra et al. (2003). We then extrapolate to the epoch of detection of GRB $040422(1.90 \mathrm{~h})$ with the reported temporal index. The result is presented in Fig. 7, which shows the afterglow of GRB 040422, after correcting for the Milky Way absorption. The error bars indicate the uncertainty of the extrapolated $K_{\mathrm{s}}$ magnitude at the time of our observation given the uncertainties in the indices given in Table 2, assuming that the power law model is valid. When there is no error reported in Table 2, we assume an error of 0.08 for the temporal index, 0.2 for the spectral index, i.e. the average of the errors listed in the table. 
Table 2. A compilation of afterglow data. References are given in superscripts. Note that the magnitude measurements are not necessarily the first available: in some cases, later but more accurate measurements were preferred.

\begin{tabular}{lllllll}
\hline \hline Name & Redshift & Temporal index & Spectral index & Magnitude & Time (h) & Host galaxy \\
\hline GRB 970228 & $0.695^{(1)}$ & $1.10 \pm 0.1^{(2)}$ & $0.61 \pm 0.32$ & $R=20.9^{(3)}$ & 20 & $R=24.6, K=22.6^{(4)}$ \\
GRB 971214 & $3.418^{(5)}$ & $1.20 \pm 0.02^{(6)}$ & $0.93 \pm 0.06^{(7)}$ & $K=18.03^{(8)}$ & 3.5 & $R=25.6, K=22.4^{(4)}$ \\
GRB 980326 & $1.0^{(9)}$ & $2 . \pm 0.1^{(9)}$ & $0.8 \pm 0.4^{(9)}$ & $R=21.25^{(9)}$ & 11.1 & $V=29.3^{(10)}$ \\
GRB 980519 & & $2.30 \pm 0.12^{(11)}$ & $1.4 \pm 0.3^{(11)}$ & $R=20.28^{(11)}$ & 15.59 & $R=26.05^{(12)}$ \\
GRB 990123 & $1.598^{(13)}$ & $1.10 \pm 0.03^{(14)}$ & $0.8 \pm 0.1^{(14)}$ & $R=18.65^{(14)}$ & 4.06 & $R=24.3, K=21.9^{(4)}$ \\
GRB 990510 & $1.619^{(15)}$ & $0.76 \pm 0.01^{(16)}$ & $0.61 \pm 0.12^{(16)}$ & $R=17.54^{(17)}$ & 3.45 & $R=27.5^{(4)}$ \\
GRB 010921 & $0.45^{(18)}$ & $1.59 \pm 0.18^{(18)}$ & $2.22 \pm 0.23^{(18)}$ & $R=19.4^{(19)}$ & 21.8 & $R=21.45, K=19.05^{(4)}$ \\
GRB 011121 & $0.36^{(20)}$ & $1.72 \pm 0.05^{(20)}$ & $0.66 \pm 0.13^{(20)}$ & $R=19.06^{(20)}$ & 10.37 & $R=24.6^{(4)}$ \\
GRB 020405 & $0.69^{(21)}$ & $1.54 \pm 0.06^{(21)}$ & $1.3 \pm 0.2^{(21)}$ & $R=20.17^{(21)}$ & 23.62 & $R=20.9^{(4)}$ \\
GRB 020813 & $1.255^{(22)}$ & $0.76 \pm 0.05^{(23)}$ & $1.04 \pm 0.03^{(23)}$ & $R=18.49^{(23)}$ & 3.97 & $R=24.7^{(4)}$ \\
GRB 021211 & $1.004^{(24)}$ & $1.11 \pm 0.01^{(25)}$ & $0.6 \pm 0.2^{(25)}$ & $R=21.9^{(25)}$ & 6.81 & $R=25.16^{(25)}$ \\
GRB 030329 & $0.1685^{(26)}$ & $0.89 \pm 0.01^{(27)}$ & $0.71^{(28)}$ & $R=15.02^{(28)}$ & 9.08 & \\
GRB 030429 & $2.658^{(29)}$ & $0.95 \pm 0.03^{(29)}$ & $0.36 \pm 0.12^{(29)}$ & $K=17.70^{(29)}$ & 13.63 & \\
GRB 030528 & & $\sim 1.2^{(30)}$ & & $K=18.6^{(30)}$ & 16.6 & $R=22.0^{(30)}$ \\
GRB 031203 & $0.1055^{(31)}$ & $\geqq 2^{(32)}$ & $2.36 \pm 0.02^{(32)}$ & $K=17.56^{(32)}$ & 9 & $K=16.5^{(32)}$ \\
GRB 040924 & $0.859^{(33)}$ & $0.7^{(34)}$ & & $K=17.5^{(35)}$ & 2.40 & $K=20.4^{(36)}$ \\
GRB 041006 & $0.712^{(37)}$ & $0.7^{(38)}$ & $0.45^{(39)}$ & $R=18.5^{(38)}$ & 1.90 & \\
\hline
\end{tabular}

(1) Bloom et al. (2001); (2) Fruchter et al. (1999); (3) Masetti et al. (1998); (4) Le Floc'h et al. (2004), and references therein; (5) Kulkarni et al. (1998); (6) Diercks et al. (1998); (7) Reichart (1998); (8) Gorosabel et al. (1998); (9) Bloom et al. (1999); (10) Chary et al. (2002); (11) Vrba et al. (2000); ${ }^{(12)}$ Sokolov et al. (1998); ${ }^{(13)}$ Hjorth et al. (1999); ${ }^{(14)}$ Kulkarni et al. (1999); ${ }^{(15)}$ Vreeswijk et al. (2001); (16) Stanek et al. (1999); (17) Harrison et al. (1999); (18) Price et al. (2002); (19) Park et al. (2001); (20) Garnavich et al. (2003); (21) Masetti et al. (2003); (22) Barth et al. (2003); (23) Covino et al. (2003); (24) Della Valle et al. (2002); (25) Pandey et al. (2003); (26) Greiner et al. (2003); (27) Torii et al. (2003); (28) Matheson et al. (2003); (29) Jakobsson et al. (2004); (30) Rau et al. (2004); (31) Prochaska et al. (2004); (32) Malesani et al. (2004b); (33) Wiersema et al. (2004); ${ }^{(34)}$ Fox (2004); (35) Terada \& Akiyama (2004); (36) from Terada et al. (2004), $27.7 \mathrm{~h}$ after the burst, includes a contribution from the afterglow; ${ }^{(37)}$ Fugazza et al. (2004); ${ }^{(38)}$ Price et al. (2004); ${ }^{(39)}$ computed from Da Costa \& Noel (2004).

A cautionary comment must be made at this point. We choose a simple modelling for the extrapolation, which could introduce biases mainly in two ways.

- The temporal curve may deviate from a power law behaviour at early times; e.g. GRB 970508 was nearly constant during the first day before decaying (Djorgovski et al. 1997), while GRB 990123 showed an optical flash was detected in the first minutes (Akerlof \& McKay 1999). Moreover, afterglows tend to have steeper temporal indices at later times (e.g. Fox et al. 2004). This is apparent in Fig. 7, where we have marked in bold the extrapolated lightcurves of afterglows observed less than six hours after the bursts (i.e. three times the delay of our observation of GRB 040422), for which we can expect a lower extrapolation error, and which are therefore more significant for our purpose.

- The absorption induces variations with wavelength of the spectral index. This can lead to overestimation of the $K_{\mathrm{s}}$ flux extrapolated from the observed $R$ magnitude that is more affected by extinction. Interestingly, in Fig. 7, six of the nine faintest points are $K_{\mathrm{s}}$ measurements, and all the nine brightest points are $R$ measurements extrapolated to the $K_{\mathrm{s}}$ band. This suggests that extinction might be playing a role for some of these GRBs. As already discussed, extinction due to the Milky Way is subject to inaccuracy, especially at low Galactic latitudes. However, the afterglow of GRB 011121 is the only case for which we made an extrapolation to the $K$ band with $A_{R}$ (Milky Way) $>1$. For GRB 021211, we have only $A_{R}$ (Milky Way) $=0.067$. Estimating the extinction within the host galaxy is beyond the scope of this paper. However, the study by Stratta et al. (2004) indicates that the reddening might be low: in the rest frame of the host, $A_{\mathrm{V}}$ (host) $=0.21 \pm 0.12$ for GRB 971214 and consistent with zero for GRB 980519, GRB 990123, and GRB 990510. For GRB 020813, Savaglio \& Fall (2004) infer a relatively high extinction of $A_{\mathrm{V}}$ (host) $\simeq 0.4$, but with weak dependence on wavelength, and therefore little impact on the power law shape of the spectrum. This suggests that, at least for the afterglows of Table 2 observed in the $R$ band, the extinction within the host might be small.

Keeping these caveats in mind, Fig. 7 shows that the afterglow of GRB 040422 appears to be significantly dimmer than most of those shown in Fig. 7. The only comparable afterglows are the ones of GRB 021211, already discussed by Pandey et al. (2003) in the context of dark bursts and 


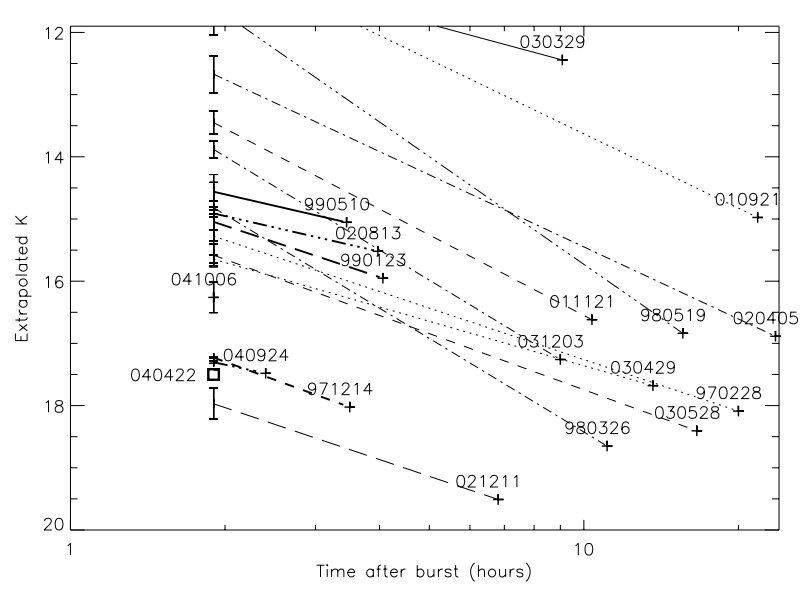

Fig. 7. Light curves of the afterglows listed in Table 2 with magnitude extrapolated to the $K_{\mathrm{s}}$ band after correction of the Milky Way absorption. The cross is placed at the time of observation. The lines indicate the temporal power-law decay backward in time up to the the time of observation of GRB 040422, different line styles are used for clarity only. Our measurement of the afterglow of GRB 040422 is indicated by a square. The error bars indicate the uncertainty of the extrapolated $K_{\mathrm{s}}$ magnitude at the time of our observations given the uncertainties on the indices in Table 2 and assuming that the power law model is valid. The extrapolated lightcurve of afterglows observed less than six hours after the bursts are marked in bold.

qualified as "not so dark", and of GRB 040924, observed 2.4 h after the burst in the $K$ band by the Subaru telescope by Terada \& Akiyama (2004) following the prompt localization in the $R$ band by the robotic Palomar 60-inch telescope (Fox \& Moon 2004). This means that if prompt observations with the VLT had not been triggered, GRB 040422 would have been classified as dark; indeed, from an observational point of view, it is optically dark and infrared faint.

This faintness contrasts with the relative brightness of the host galaxy. Less than two hours after the burst, it is just 2.3 mag fainter than the afterglow. In Table 2 we give the magnitudes of the hosts for our sample. Again using the temporal and spectral indices, we extrapolate the magnitude of the afterglow in the band in which the magnitude of the host is given, $1.90 \mathrm{~h}$ after the trigger. We find that on the average the afterglow is 7 mag brighter than its host, this figure still holds if we restrain the sample to the 6 GRBs for which the magnitude of the host is given in the $K$ band. For only two GRBs the difference is close to the one of GRB $0404221.90 \mathrm{~h}$ after the burst: GRB 040924 (difference $3 \mathrm{mag}$ ) and the very extreme case of GRB 031203 (difference $2.3 \mathrm{mag}$, actually the afterglow was fainter than the host at the time of observation), which also has a very high spectral index, $\beta=2.37$, to be compared to the high values we derived in Sect. 3. This indicates that GRB 040422 may share some properties with GRB 031203 with respect to the environment of the burst and its host galaxy.

These results show that $8-\mathrm{m}$ class telescopes operated in the infrared have the capability of exploring the dark/faint burst domain, therefore reducing the proportion of burst that is considered dark only because of inefficient follow-up. Indeed, we may consider the possibility that dark bursts do not really exist and that their afterglows are simply fainter with different global spectral properties. Another possibility is that we are observing an afterglow enshrouded by dust surrounding the burst site, although so far it seems that no absorption is the rule. For GRB 040422, neither of these possibilities can be excluded. More information could have been gathered by acquiring a spectrum, which would have required quick localization by a robotic telescope. REM (Zerbi et al. 2002) has now the ability to promptly localize such an event: if we assume a temporal index of -1 , the afterglow of GRB 040422 would have $K_{\mathrm{s}}=14.6$, 5 min after the burst, corresponding to the $5 \sigma$ detection limit of REM with a 30-s exposure. In 2008, the X-shooter spectrograph (Moorwood \& d'Odorico 2004) will be operational at the VLT and sensitive enough to obtain the spectrum of an afterglow similar to the one of GRB 040422 in the infrared and in the optical with medium resolution (5000-8000 in the infrared), provided that the observation is as rapid as in the case of GRB 040422. With such a spectrum, it will be possible to measure the redshift, to quantify the reddening precisely, and to study the properties of the host galaxy in a unique and comprehensive way, thus increasing our understanding of the GRB environment.

\section{Conclusion}

We have described the results obtained on GRB 040422 using IBIS and SPI on the INTEGRAL satellite and the instruments FORS 2 and ISAAC on the VLT. The main results are:

- the IBIS spectrum is well-fit with the Band model;

- only two hours after the trigger, the afterglow was below the detection limit of the 2 MASS catalog, therefore a second epoch observation was required to spot the afterglow;

- we detected the afterglow in the $K_{\mathrm{S}}$ band, but not in the $R$ and $I$ band, at least partially because of the Milky Way absorption;

- we detected the bright host galaxy in the $K_{\mathrm{s}}$ band, with $K_{\mathrm{s}}=20.3 \pm 0.2$ to be corrected from a 0.5 mag Milky Way absorption;

- comparison with a compilation of quickly observed afterglows indicates that the one of GRB 040422 is the dimmest, with the exception of the afterglow of GRB 021211 and possibly GRB 040924.

Without our prompt infrared observations, GRB 040422 would probably have been classified as dark. It is nevertheless faint in the infrared. This suggests that the proportion of dark GRBs can be significantly lowered by a more systematic use of 8-m class telescopes in the infrared in the very first hours after the burst. Quicker robotic telescopes operating in the infrared, like REM, and the faster and more precise positions that will be provided soon by Swift will of course facilitate the identification, allowing spectrophotometric observations to explore the domain of dim afterglows.

Acknowledgements. It is a pleasure to thank the ESO staff at the VLT for the observations. Data mining for constructing Table 2 was speeded up by the use of Jochen Greiner's GRB page http://www . mpe.mpg.de/ jcg/grb.html. We thank D. Lazzati, L. Guzzo, S. Bhargavi, and P. Véron for useful discussions and 
comments. P.F. wishes to thank the CNRS/APC for funding and B. Bigot, Haut Commissaire à l'Énergie Atomique, for funding and support. DM wishes to thank the Italian Istituto Nazionale di Astrofisica (INAF).

\section{References}

Akerlof, C. W., \& McKay, T. A. 1999, GCN, 205

Band, D. L., Matteson, J., Ford, L., et al. 1993, ApJ, 413, 281

Barth, A. J., Sari, R., Marshall, H. C., et al. 2003, ApJ, 548, 47

Berger, E., Kulkarni, S. R., Bloom, J. S., et al. 2002, ApJ, 581, 981

Bertin, E., \& Arnouts, S. 1996, A\&A, 117, 393

Bloom, J. S., Kulkarni, S. R., Djorgovski, S. G., et al. 1999, Nature, 401,453

Bloom, J. S., Djorgovski, S. G., \& Kulkarni, S. R. 2001, ApJ, 554, 678

Cardelli, J. A., Clayton, G. C., \& Mathis, J. S. 1989, ApJ, 345, 245

Chary, R., Becklin, E. E., \& Armus, L. 2002, ApJ, 556, 229

Collinge, M. J., Strauss, M. A., Hall, P. B., et al. 2004 [arXiv: astro-ph/0411620]

Courvoisier, T. J. L., Walter, R., Beckmann, V., et al. 2003, A\&A, 411, L53

Covino, S., Malesani, D., Tavecchio, F., et al. 2003, A\&A, 404, L5

Da Costa, G., \& Noel, N. 2004, GCN, 2789

Della Valle, M., Benetti, S., Malesani, D., et al. 2003, A\&A, 406, L33

Dickey, J., \& Lockman, F. J. 1990, ARA\&A, 28, 215

Diercks, A. H., Deutsch, E. W., Castander, F. J., et al. 1998, ApJ, 503, 105

Djorgovski, S. G., Metzger, M. R., Kulkarni, S. R., et al. 1997, Nature, 387,876

Dutra, C. M., Ahumada, A. V., Clariá, J. J., et al. 2003, A\&A, 408, 287

Fiore, F., d'Elia, V., Lazzati, D., et al. 2004 [arXiv: astro-ph/0409717]

Fishman, G. J., \& Meegan, C. A. 1995, ARA\&A, 33, 415

Fox, D. B., \& Moon, D. S. 2004, GCN, 2734

Fox, D. B. 2004, GCN, 2741

Fox, D. B., Yost, S., Kulkarni, S. R., et al. 2003, Nature, 422, 284

Fruchter, A., Pian, E., Thorsett, S., et al. 1999, ApJ, 516, 683

Fugazza, D., Fiore, F., Covino, S., et al. 2004, GCN, 2782

Fynbo, J. U., Jensen, B. L., Gorosabel, J., et al. 2001, A\&A, 369, 373

Garnavich, P. M., Stanek, K. Z., Wyrzykowski, L., et al. 2003, ApJ, 582,924

Gorosabel, J., Castro-Tirado, A. J., Willott, C. J., et al. 1998, A\&A, 335, L8

Götz, D., Mereghetti, S., Hurley, K., et al. 2003, A\&A, 409, 831

Götz, D., Mereghetti, S., Produit, N., \& Mowlawi, N. 2004, GCN, 2695

Graham, J. A. 1982, PASP, 94, 244

Greiner, J., Peimbert, M., Esteban, C., et al. 2003, GCN, 2020

Harrison, F. A., Bloom, J. S., Frail, D. A., et al. 1999, ApJ, 523, L121

Hjorth, J., Andersen, M. I., Pedesen, H., et al. 1999, GCN, 249

Jakobsson, P., Hjorth, J., Fynbo, J. U., et al. 2004, A\&A, 427, 785

Kulkarni, S. R., Djorgoski, S. G., Ramaprakash, A. N., et al. 1998, Nature, 393, 35

Kulkarni, S. R., Djorgovski, S. G., Odewahn, S. C., et al. 1999, Nature, 398, 389

Lamb, D. Q. 2000, Phys. Rep., 505, 333

Lamb, D. Q., \& Reichart, D. E. 2000, ApJ, 536, 1

Lamb, D. Q., Ricker, J. R., Atteia, J.-L., et al. 2004, New A Rev., 48, 423

Lazzati, D., Covino, S., \& Ghisellini, G. 2002, MNRAS, 330, 583

Lebrun, F., Leray, J. P., Lavocat, P., et al. 2003, A\&A, 411, L141

Le Floc'h, E., Duc, P.-A., Mirabel, I. F., et al. 2004, A\&A, 400, 490
McBreen, S., McBreen, B., Quilligan, F., \& Hanlon, L. 2002, A\&A, 385, L19

Malesani, D., Fugazza, D., Ghirlanda, G., et al. 2004a, GCN, 2573

Malesani, D., Tagliaferri, G., Chincarini, G., et al. 2004b, ApJ, 609, 5

Malesani, D., D'Avanzo, P., Melandri, A., et al. 2004c, GCN, 2685

Masetti, N., Bartolini, C., Guarnieri, A., \& Piccioni, A. 1998, Nucl. Phys. B Proc. Suppl., 69, 674

Masetti, N., Palazzi, E., Pian, E., et al. 2003, A\&A, 404, 465

Matheson, T., Garnavich, P. M., Stanek, K. Z., et al. 2003, ApJ, 599, 394

Mereghetti, S., Götz, D., Borkowski, J., Walter, R., \& Pedersen, H. 2003, A\&A, 411, L291

Mereghetti, S., Götz, D., Borkowski, J., et al. 2004a, in Proc. 5th INTEGRAL Workshop: The INTEGRAL Universe (Munich), ESA Special Publication SP-552, ed. V. Schnfelder, G. Lichti, \& C. Winkler [arXiv: astro-ph/0404019]

Mereghetti, S., Gotz, D., Borkowski, J., \& Beck, M. 2004b, GCN, 2572

Mereghetti, S., Götz, D., Andersen, M. I., et al. 2004c [arXiv: astro-ph/0412012]

Moorwood, A., \& d'Odorico, S. 2004, The ESO Messenger, 115, 8

Moran, L., Mereghetti, S., Götz, D., et al. 2005, A\&A, 432, 467

Pandey, S. B., Anupama, G. C., Sagar, R., et al. 2003, A\&A, 408, L21

Park, H. S., Williams, G., Barthelmy, S., et al. 2001, GCN, 1131

Persson, S. E., Murphy, D. C., Krzeminski, W., Roth, M., \& Rieke, M. J. 1998, ApJ, 116, 2475

Predehl, P., \& Schmitt, J. H. M. M. 1995, A\&A, 293, 889

Price, P. A., Kulkarni, S. R., Berger, E., et al. 2002, ApJ, 571, 121

Price, P. A., Da Costa, G., \& Noel, N. 2004, GCN, 2771

Prochaska, J. X., Bloom, J. S., Chen, H., et al. 2004, ApJ, 611, 200

Quilligan, F., McBreen, B., Hanlon, L., et al. 2002, A\&A, 385, 377

Rau, A., Greiner, J., Klose, S., et al. 2004, A\&A, 427, 815

Reichart, D. E. 1998 [arXiv: astro-ph/9801139]

Reichart, D. E., \& Price, P. A. 2002, ApJ, 565, 174

Rykoff, E. 2004, GCN, 2576

Savaglio, S., \& Fall, S. M. 2004, ApJ, 614, 293

Sazonov, S. Y., Lutovinov, A. A., \& Sunyaev, R. A. 2004, Nature, 430, 646

Schlegel, D., Finkbeiner, D., \& Davis, M. 1998, ApJ, 500, 525

Skinner, G., \& Connell, P. 2003, A\&A, 411, L123

Skrutskie, M. F. 2001, BAAS, 33, 827

Soderberg, A. M., Kulkarni, S. R., Berger, E., et al. 2004, Nature, 430, 648

Sokolov, V., Zharikov, S., Palazzi, E., et al. 1998, GCN, 148

Stanek, K. Z., Garnavich, P. M., Kaluzny, J., et al. 1999, ApJ, 522, 39

Stratta, G., Fiore, F., Antonelli, L. A., et al. 2004, ApJ, 608, 846

Tagliaferri, G., Fugazza, D., Covino, S., et al. 2004, GCN, 2535

Terada, H., \& Akiyama, M. 2004, GCN, 2742

Terada, H., Akiyama, M., \& Kawai, N. 2004, GCN, 2750

Torii, K., Kato, T., Yamaoka, H., et al. 2003, ApJ, 597, 101

Ubertini, P., Lebrun, F., Di Cocco, G., et al. 2003, A\&A, 411, L131

Ulrich, M.-H., Maraschi, L., \& Urry, C. M. 1997, ARA\&A, 35, 445

van Paradjis, J., Kouveliotou, C., \& Wijers, R. 2000, ARA\&A, 38, 379

Vaughan, S., Willingale, R., O'Brien, P. T., et al. 2004, ApJ, 603, 5

Vedrenne, G., Roques, J. P., Schönfelder, V., et al. 2003, A\&A, 411, L63

von Kienlin, A., Beckmann, V., Rau, A., et al. 2003, A\&A, 411, L299

Vrba, F. J., Henden, A. A., Canzian, B., et al. 2000, ApJ, 528, 254

Vreeswijk, P. M., Fruchter, A., Kaper, L., et al. 2001, ApJ, 546, 672

Watson, D., Hjorth, J., Levan, A., et al. 2004, ApJ, 605, 101

Wiersema, K., Starling, R., Rol, E., et al. 2004, GCN, 2800

Winkler, C., Courvoisier, T. J., Di Cocoo, G., et al. 2003, A\&A, 411, L1

Zerbi, F. M., Chincarini, G., Ghisellini, G., et al. 2002, Astron. Nachr., 322, 275 